\title{
A priori bounds for a class of semi-linear degenerate elliptic equations
}

\author{
Genggeng Huang*
}

(School of Mathematics Sciences, LMNS, Fudan University, Shanghai, China, 200433)

\begin{abstract}
In this paper, we mainly discuss a priori bounds of the following degenerate elliptic equation,$$
a^{i j}(x) \partial_{i j} u+b^{i}(x) \partial_{i} u+f(x, u)=0, \text { in } \Omega \subset \subset R^{n},
$$

where $a^{i j} \partial_{i} \phi \partial_{j} \phi=0$ on $\partial \Omega, \phi$ is the defining function of $\partial \Omega$. Imposing suitable conditions on the coefficients and $f(x, u)$, one can get the $L^{\infty}$-estimates of 0.1 via blow up method.
\end{abstract}

Key Words: degenerate elliptic, characteristic, semi-linear elliptic

MSC2010: 35J15, 35J61, 35J70

\section{Introduction}

In the study of the existence and regularity of the elliptic equations, a priori estimates play an important role at least as far as Schauder's work in 1930s. For the uniformly linear elliptic equations, one can get the $L^{\infty}$-estimates using the barrier function. But for non-linear elliptic equations, this method fails. In [6], the authors use a scaling argument reminiscent to that used in the theory of Minimal Surfaces to get the a priori estimates for some uniformly semi-linear elliptic equations. This inspires us to consider the degenerate case. We mainly consider a priori bounds of the following degenerate elliptic equation which arises from the study of isometric embedding,

$$
a^{i j}(x) \partial_{i j} u+b^{i}(x) \partial_{i} u+f(x, u)=0, \text { in } \Omega \subset \subset R^{n} .
$$

Let $\phi \in C^{2}$ be the defining function of $\partial \Omega$, namely,

$$
\left.\phi\right|_{\partial \Omega}=0,\left.\nabla \phi\right|_{\partial \Omega} \neq 0, \phi>0 \text { in } \Omega \cap \mathcal{N}(\partial \Omega)
$$

where $\mathcal{N}(\partial \Omega)$ is a neighborhood of $\partial \Omega$. Also, suppose that

$$
a^{i j} \partial_{i} \phi \partial_{j} \phi=0, \nabla\left(a^{i j} \partial_{i} \phi \partial_{j} \phi\right) \neq 0 \text { on } \partial \Omega \in C^{2}
$$

and that for the eigenvalues of $\left\{a^{i j}\right\} \lambda_{1}, \ldots, \lambda_{n}$, there hold, for some constant $c_{0}$,

$$
\lambda_{1}, \ldots, \lambda_{n-1} \geq c_{0}>0, \lambda_{n}=m(x) \phi, 0<m(x) \in C(\bar{\Omega}) .
$$

Theorem 1.1. Let (1.2), (1.3) and (1.4) be fulfilled. Suppose that $0<u \in C^{2}(\Omega) \cap L^{\infty}(\Omega)$ solves (1.1) and that $a^{i j} \in C^{2}(\bar{\Omega}), b^{i} \in C^{1}(\bar{\Omega})$ and $f(x, t) \in C(\bar{\Omega} \times[0, \infty))$

$$
\lim _{t \rightarrow \infty} \frac{f(x, t)}{t^{\alpha}}=h(x), \text { uniformly for some } 1<\alpha<\frac{n+2 a+1}{n+2 a-3},
$$

*genggenghuang@fudan.edu.cn 
where $0<h(x) \in C(\bar{\Omega})$,

$$
a=\sup _{\partial \Omega} \frac{b^{i} \partial_{i} \phi-\partial_{j} a^{i j} \partial_{i} \phi}{\partial_{k} a^{i j} \partial_{i} \phi \partial_{j} \phi \phi^{k}}+1, b \triangleq \inf _{\partial \Omega} \frac{b^{i} \partial_{i} \phi-\partial_{j} a^{i j} \partial_{i} \phi}{\partial_{k} a^{i j} \partial_{i} \phi \partial_{j} \phi \phi^{k}}>\frac{1}{2}, \phi^{k}=\frac{\partial_{k} \phi}{|\nabla \phi|^{2}} .
$$

Then it follows that

$$
|u|_{L^{\infty}} \leq C \text { for some constant } C \text { independent of } u .
$$

Remark 1.1. Define

$$
g(x)=\frac{b^{i} \partial_{i} \phi-\partial_{j} a^{i j} \partial_{i} \phi}{\partial_{k} a^{i j} \partial_{i} \phi \partial_{j} \phi \phi^{k}} \text { on } \partial \Omega \text { where } a^{i j} \partial_{i} \phi \partial_{j} \phi=0 .
$$

The invariance of $g(x)$ is proved in [7]. The numerator of $g(x)$ is the well-know Fichera number. The concept of Fichera number is very important when we deal with degenerate elliptic problems with boundary characteristic degenerate. It indicates whether we shall impose boundary condition in such case. This fact was first observed by M.V.Keldyš in [10] and developed by Fichera in [2, [3]. The Fichera number also affects the regularities of the solutions up to the boundary, see [7]. For more details of Fichera number, refer to [11].

For dimension $n=2$, Theorem 1.1] was proved in [9. The main difficulties arise from the degeneracy on the boundary and no boundary condition. In 9], we establish regularity results up to the boundary to overcome the difficulties. The method used in [9] can't improve the regularity for $n \geq 3$. We use De Giorgi-Moser method to get the Hölder regularity on the boundary. It should be emphasized that we didn't get the uniform Hölder regularity in $\bar{\Omega}$ but on $\partial \Omega$.

Also when we consider one of the blow up equation,

$$
y u_{y y}+a u_{y}+\Delta_{x} u+u^{\alpha}=0 \text { in } R_{+}^{n}, u \in L_{l o c}^{\infty}\left(\overline{R_{+}^{n}}\right) .
$$

During the process of blow up, we know that we can only expect $L^{\infty}$-regularity of blow up solution $u$ in (1.8). As the Liouville type theorem in [9] was obtained under the assumption $u \in C^{2}\left(\overline{R_{+}^{n}}\right)$. Thus we must improve the regularity up to the boundary for (1.8). By carefully deal with the boundary and the energy inequality, we improved the regularity. Hence, we must have the following two theorem to overcome the difficulties.

Theorem 1.2. Let (1.2), (1.3) and (1.4) be fulfilled. Moreover, $g(x)>0$ on the boundary $\partial \Omega$ which is defined in Remark 1.1. Suppose that $0<u \in C^{2}(\Omega) \cap L^{\infty}(\Omega)$ solves (1.1) and that $a^{i j} \in C^{2}(\bar{\Omega}), b^{i} \in C^{1}(\bar{\Omega})$ and $f(x, t) \in C(\bar{\Omega} \times[0, \infty))$. Then:

$$
|u(x)-u(y)| \leq C|x-y|^{\beta}, \forall x \in \bar{\Omega}, y \in \partial \Omega, \text { for some } \beta \in(0,1),
$$

where $C, \beta$ depends only on $|u|_{\infty},\left|a^{i j}\right|_{C^{2}},\left|b^{i}\right|_{C^{1}},|\partial \Omega|_{C^{2}},|f|_{C^{0}}$ and $n$.

Theorem 1.3. Let $u \geq 0$ solves (1.8) with $a>\frac{3}{2}, \alpha \geq 1$. Then $u \in C^{2}\left(\overline{R_{+}^{n}}\right)$.

The present paper organizes as the following. In Section 2, by the De Giorgi-Moser method we get the Hölder regularity on the boundary of (1.4). In Section 3, the utility of energy integral helps us improve the regularity. In Section 4, we prove Theorem 1.1 via the blow up method.

\section{$2 C^{\alpha}$ regularity on the boundary}

This section is devoted to the proof of Theorem 1.2 The proof is based on the De Giorgi-Moser method. We first derive a weighted-Sobolev inequality for later use. Denote by $\widetilde{W}^{1,2}(G)$ where $G \subset R_{+}^{n}=\left\{\left(x_{1}, \ldots x_{n}\right) \in R^{n} \mid x_{n}>0\right\}$ the completion of all the functions $u \in C^{1}(\bar{G})$ under the following norm

$$
\left(\int_{G}\left(x_{n}\left(\partial_{n} u\right)^{2}+\left|\nabla_{x^{\prime}} u\right|^{2}+u^{2}\right) d x\right)^{\frac{1}{2}}
$$


Lemma 2.1. (1)For all $u \in \widetilde{W}^{1,2}(G)$ with $u=0$ on $\partial G \cap R_{+}^{n}$ there is a universal constant $C$ independent of $G$ such that

$$
\left(\int_{G}|u|^{\frac{2(n+1)}{n-1}} d x\right)^{\frac{n-1}{n+1}} \leq C \int_{G}\left(x_{n}\left(\partial_{n} u\right)^{2}+\left|\nabla_{x^{\prime}} u\right|^{2}\right) d x .
$$

(2) With $G_{1}=\left\{0<x_{n}<1,\left|x^{\prime}\right|^{2}<1\right\}$ for any $\epsilon>0$ there exists a constant $C_{\epsilon}$ such that

$$
\int_{G_{1}} u^{2} d x \leq C_{\epsilon} \int_{G_{1}}\left(x_{n}\left(\partial_{n} u\right)^{2}+\left|\nabla_{x^{\prime}} u\right|^{2}\right) d x
$$

for all $u \in C^{1}\left(\bar{G}_{1}\right)$ subject to $\left|\left\{x \in G_{1} \mid u(x)=0\right\}\right| \geq \epsilon$.

Proof. The proof for the present lemma is based on the raising dimension argument. Define a transformation

$$
T: \quad G \ni\left(x^{\prime}, x_{n}\right) \longrightarrow\left(x^{\prime}, 2 \sqrt{x_{n}}\right)=\left(x^{\prime}, y\right) \in T(G)
$$

Let $G \subset R_{+}^{n}$ and $u \in \widetilde{W^{1,2}}(G)$. Lift $T(G)$ in $R^{n+1}$ by such a way $\widetilde{T(G)}=\left\{\left(x^{\prime}, y, z\right) \in R^{n+1} \mid\left(x^{\prime}, y\right) \in\right.$ $T(G), 0<z<y\}$. Then

$$
\int_{G}|u|^{p} d x=\frac{1}{2} \int_{T(G)}\left|u \circ T^{-1}\right|^{p} y d x^{\prime} d y=\frac{1}{2} \|\left. u \circ T^{-1}\right|_{L^{p}(\widetilde{T((G))})} ^{p}
$$

and

$$
\int_{G}\left(x_{n}\left(\partial_{n} u\right)^{2}+\left|\nabla_{x^{\prime}} u\right|^{2}\right) d x=\frac{1}{2} \int_{T(G)}\left|\nabla_{x^{\prime}, y}\left(u \circ T^{-1}\right)\right|^{2} y d x^{\prime} d y=\frac{1}{2}\left\|\widetilde{\nabla}\left(u \circ T^{-1}\right)\right\|_{L^{2}(\widetilde{T(G)})}^{2}
$$

where $\widetilde{\nabla}=\left(\nabla_{x^{\prime}}, \partial_{y}, \partial_{z}\right)$ is the gradient in $R^{n+1}$. Now let us deal with the first inequality of Lemma 2.1. It suffices to prove (2.1) is valid for all $u \in C^{1}(\bar{G})$. Let $u \in C^{1}(\bar{G})$ with $u=0$ at $\partial G \cap R_{+}^{n}$. Set $\tilde{u}=u$ as $\left(x^{\prime}, x_{n}\right) \in G$ and $\tilde{u}=0$ as $\left(x^{\prime}, x_{n}\right) \in R_{+}^{n} \backslash G$,

$$
P\left(u \circ T^{-1}\right)\left(x^{\prime}, y, z\right)= \begin{cases}\tilde{u} \circ T^{-1}\left(x^{\prime}, y, z\right) & \text { in } \widetilde{T(G)} \\ \tilde{u} \circ T^{-1}\left(x^{\prime}, z, y\right) & \text { as } z>y>0 .\end{cases}
$$

Then we can extend $P\left(u \circ T^{-1}\right)$ to $R^{n+1}$ by symmetric extension first with respect to the plan $y=0$ and then to the plan $z=0$. Obviously, $P\left(u \circ T^{-1}\right) \in H^{1}\left(R^{n+1}\right) \hookrightarrow L^{q}\left(R^{n+1}\right)$ where $q=\frac{2(n+1)}{n-1}$. Therefore by (2.3), (2.4) and the standard Sobolev embedding theorem it turns out

$$
\begin{aligned}
\left(\int_{G}|u|^{\frac{2(n+1)}{n-1}} d x\right)^{\frac{n-1}{n+1}} & =\frac{1}{8}\left(\int_{R^{n+1}}\left|P\left(u \circ T^{-1}\right)\right|^{\frac{2(n+1)}{n-1}} d x^{\prime} d y d z\right)^{\frac{n-1}{n+1}} \\
& \leq C\left\|\widetilde{\nabla}\left(P\left(u \circ T^{-1}\right)\right)\right\|_{L^{2}\left(R^{n+1}\right)}^{2} \\
& =8 C \int_{\widetilde{T(G)}}\left|\widetilde{\nabla}\left(P\left(u \circ T^{-1}\right)\right)\right|^{2} d x^{\prime} d y d z \\
& =16 C \int_{G}\left(x_{n}\left(\partial_{n} u\right)^{2}+\left|\nabla_{x^{\prime}} u\right|^{2}\right) d x
\end{aligned}
$$

for some universal constant $C$ independent of $u$.

Now let us deal with (2.2) which corresponds to the Poincare inequality. Suppose that $u \in$ $C^{1}\left(\bar{G}_{1}\right)$ where $G_{1}=\left\{0<x_{n}<1,\left|x^{\prime}\right|^{2}<1\right\}$, subject to $\left|\left\{\left(x^{\prime}, x_{n}\right) \in G_{1} \mid u\left(x^{\prime}, x_{n}\right)=0\right\}\right| \geq \epsilon>0$. Then $\left|\left\{\left(x^{\prime}, y, z\right) \in \widehat{T\left(G_{1}\right)} \mid\left(u \circ T^{-1}\right)\left(x^{\prime}, y, z\right)=0\right\}\right| \geq \epsilon / C$ for some unversal constant $C$. Hence using the well-known Poincare inequality we can get

$$
\int_{G_{1}}|u|^{2} d x=\frac{1}{2} \int_{\widetilde{T\left(G_{1}\right)}}\left|u \circ T^{-1}\right|^{2} d x^{\prime} d y d z
$$




$$
\begin{aligned}
& \leq C_{\epsilon} \int_{\widetilde{T\left(G_{1}\right)}}\left|\widetilde{\nabla}\left(u \circ T^{-1}\right)\right|^{2} d x^{\prime} d y d z \\
& =2 C_{\epsilon} \int_{G_{1}}\left(x_{n}\left(\partial_{n} u\right)^{2}+\left|\nabla_{x^{\prime}} u\right|^{2}\right) d x
\end{aligned}
$$

for some constant $C_{\epsilon}$ depending only on $\epsilon$. This completes the proof for the present lemma.

Set $G_{R}\left(x_{0}\right)=\left\{\left|x_{i}-\left(x_{0}\right)_{i}\right|<\sqrt{R}, i=1, \ldots, n-1,0<x_{n}<R\right\}$ for $x_{0}=\left(x_{0}^{\prime}, 0\right)$. Sometimes, if no confusion occurs, simply write $G_{R}$.

Consider

$$
\left\{\begin{array}{l}
\mathscr{L}(v)=\partial_{i}\left(a^{i j} \partial_{j} v\right)+b^{j} \partial_{j} v=f \in L^{\infty} \text { in } G_{R} \\
v=0 \text { on } \partial G_{R} \cap\left\{x_{n}>0\right\}
\end{array}\right.
$$

The coefficients satisfy that

$$
\left(a^{i j}\right)_{n \times n} \geq 0,\left(a^{i j}\right)_{n-1 \times n-1} \geq \lambda I \text { for some constant } \lambda>0, a^{i n}=x_{n} b^{i n}, \text { with } b^{n n}>0 .
$$

Moreover, we assume

$$
a^{i j} \in C^{2}\left(\overline{R_{+}^{n}}\right), b^{i n}, b^{i} \in C^{1}\left(\overline{R_{+}^{n}}\right), b^{n} \geq 0
$$

Lemma 2.2. If the assumptions in (2.6) and (2.7) are satisfied. Then (2.5) admits a solution $v \in H^{1}\left(G_{R}\right) \cap W_{\text {loc }}^{2, l}\left(G_{R}\right), \forall l \in[2, \infty)$, moreover, there exists $R_{0}$, such that for $\forall R \in\left(0, R_{0}\right)$, it is valid that

$$
|v|_{\infty} \leq C|f|_{\infty} R \text {, for some universal constant } C .
$$

Proof. First we shall compute the sign-invariable $D$ which is Fichera number like refer to [7] on $x_{n}=0$ where $\phi(x)=x_{n}$ :

$$
D=b^{i} \partial_{i} \phi+\frac{1}{2} \partial_{k} a^{i j} \partial_{i} \phi \partial_{j} \phi \partial_{k} \phi|\nabla \phi|^{-2}=b^{n}+\frac{1}{2} b^{n n}>0
$$

From [7, one can get the existence of a solution $v$ such that $v \in H^{1}\left(G_{R}\right)$. And by the nondegeneracy in the interior of $G_{R}, v \in W_{l o c}^{2, l}\left(G_{R}\right)$ follows immediately. Set $\bar{v}=(v-k)^{+}$. Multiplying both sides of (2.5) by $-\bar{v}$ and integrating them on $G_{R} \cap\left\{x_{n} \geq \epsilon\right\}$, one can see that

$$
\begin{aligned}
& -\int_{G_{R} \cap\left\{x_{n} \geq \epsilon\right\}} f \bar{v} \\
= & \int_{G_{R} \cap\left\{x_{n}=\epsilon\right\}} a^{j n} \bar{v} \partial_{j} \bar{v}+\int_{G_{R} \cap\left\{x_{n} \geq \epsilon\right\}} a^{i j} \partial_{i} \bar{v} \partial_{j} \bar{v}-\sum_{1}^{n-1} \int_{G_{R} \cap\left\{x_{n} \geq \epsilon\right\}} b^{j} \partial_{j} \bar{v} \bar{v} \\
+ & \frac{1}{2} \int_{G_{R} \cap\left\{x_{n}=\epsilon\right\}} b^{n} \partial_{n} \bar{v}^{2}+\frac{1}{2} \int_{G_{R} \cap\left\{x_{n} \geq \epsilon\right\}} \partial_{n} b^{n} \bar{v}^{2} \\
\geq & c_{1} \int_{G_{R} \cap\left\{x_{n} \geq \epsilon\right\}}\left(x_{n}\left|\partial_{n} \bar{v}\right|^{2}+\left|\nabla_{x^{\prime}} \bar{v}\right|^{2}\right)-c_{2} \int_{G_{R} \cap\left\{x_{n} \geq \epsilon\right\}} \bar{v}^{2}+\int_{G_{R} \cap\left\{x_{n}=\epsilon\right\}} a^{j n} \bar{v} \partial_{j} \bar{v}
\end{aligned}
$$

In getting the last inequality, we have used $b^{n} \geq 0$ and

$$
a^{i j} \xi_{i} \xi_{j} \geq c_{0}\left(x_{n} \xi_{n}^{2}+\left|\xi^{\prime}\right|^{2}\right) \text {, if } R \text { is small enough }
$$

Claim: we can choose suitable $\epsilon_{k} \rightarrow 0$ such that

$$
\int_{G_{R} \cap\left\{x_{n}=\epsilon_{k}\right\}} a^{j n} \bar{v} \partial_{j} \bar{v} \rightarrow 0
$$


As $\bar{v} \in L^{2}\left(G_{R}\right)$, without loss of generality we may assume that

$$
\int_{\bar{G}_{R} \cap\left\{x_{n}=0\right\}} \bar{v}^{2} \leq c
$$

Then

$$
\begin{aligned}
& \bar{v}\left(x^{\prime}, x_{n}\right)=\int_{0}^{x_{n}} \partial_{n} \bar{v}\left(x^{\prime}, s\right) d s+\bar{v}\left(x^{\prime}, 0\right) \\
\Rightarrow \quad & \left\|\bar{v}\left(x^{\prime}, x_{n}\right)\right\|_{L^{2}\left(G_{R} \cap\left\{x_{n}=t\right\}\right)} \leq c\left(\left\|\partial_{n} \bar{v}\right\|_{L^{2}\left(G_{R}\right)}+\left\|\bar{v}\left(x^{\prime}, 0\right)\right\|_{L^{2}\left(\bar{G}_{R} \cap\left\{x_{n}=0\right\}\right)}\right)
\end{aligned}
$$

By now, we have proved that $\bar{v}$ is uniformly integrable on each $x_{n}$-section of $G_{R}$. If $\forall \epsilon>0$, $\exists c_{0}>0$ such that

$$
\begin{gathered}
\int_{G_{R} \cap\left\{x_{n}=\epsilon\right\}} \epsilon\left|\partial_{j} \bar{v}\right|^{2} \geq c_{0} \\
\Rightarrow \quad \int_{G_{R}}\left|\partial_{j} \bar{v}\right|^{2} \geq \int_{0}^{R} \frac{c_{0}}{x_{n}} d x_{n}=\infty
\end{gathered}
$$

which is a contradiction. This means $\exists \epsilon_{k} \rightarrow 0$ such that

$$
\int_{G_{R} \cap\left\{x_{n}=\epsilon_{k}\right\}} \epsilon_{k}\left|\partial_{j} \bar{v}\right|^{2} \rightarrow 0
$$

Combining (2.11) with (2.13), one can get

$$
\left|\int_{G_{R} \cap\left\{x_{n}=\epsilon_{k}\right\}} a^{j n} \bar{v} \partial_{j} \bar{v}\right| \leq c \epsilon_{k}^{\frac{1}{2}}\left(\int_{G_{R} \cap\left\{x_{n}=\epsilon_{k}\right\}} \epsilon_{k}\left|\partial_{j} \bar{v}\right|^{2} d x^{\prime}\right)^{\frac{1}{2}}\left(\int_{G_{R} \cap\left\{x_{n}=\epsilon_{k}\right\}}|\bar{v}|^{2} d x^{\prime}\right)^{\frac{1}{2}} \rightarrow 0
$$

Thus, the claim is proved. This allows us to take $\epsilon=0$ such that (2.8) remains true with the last term vanished. It follows that

$$
\int_{G_{R}} x_{n}\left|\partial_{n} \bar{v}\right|^{2}+\left|\nabla_{x^{\prime}} \bar{v}\right|^{2} \leq c \int_{G_{R}}|f| \bar{v}+|\bar{v}|^{2}
$$

By Lemma 2.1 and inequality (2.15), we see

$$
\begin{aligned}
\left(\int_{G_{R}} \bar{v}^{\frac{2(n+1)}{n-1}}\right)^{\frac{n-1}{n+1}} & \leq c \int_{G_{R}}\left(x_{n}\left|\partial_{n} \bar{v}\right|^{2}+\left|\nabla_{x^{\prime}} \bar{v}\right|^{2}\right) \\
& \leq c\left(\int \bar{v}^{\frac{2(n+1)}{n-1}}\right)^{\frac{n-1}{n+1}}|A(k)|^{\frac{2}{n+1}}+|f|_{\infty}\left(\int \bar{v}^{\frac{2(n+1)}{n-1}}\right)^{\frac{n-1}{2(n+1)}}|A(k)|^{\frac{n+3}{2(n+1)}} \\
& \leq c\left(\int \bar{v}^{\frac{2(n+1)}{n-1}}\right)^{\frac{n-1}{n+1}}|A(k)|^{\frac{2}{n+1}}+\frac{1}{2}\left(\int \bar{v}^{\frac{2(n+1)}{n-1}}\right)^{\frac{n-1}{n+1}}+c|f|_{\infty}^{2}|A(k)|^{\frac{n+3}{n+1}}
\end{aligned}
$$

where $A_{k}=\left\{x \in G_{R} \mid v \geq k\right\}$. This implies that

$$
\begin{gathered}
(h-k)^{2}|A(h)|^{\frac{n-1}{n+1}} \leq c|f|_{\infty}^{2}|A(k)|^{\frac{n+3}{n+1}} \\
|A(h)| \leq \frac{c^{\frac{n+1}{n-1}}|f|^{\frac{2(n+1)}{n-1}}}{(h-k)^{\frac{2(n+1)}{n-1}}}|A(k)|^{\frac{n+3}{n-1}}
\end{gathered}
$$

if we take $k \geq k_{0}$ such that $c \mid A\left(k_{0}\right)^{\frac{2}{n+1}} \leq \frac{1}{4}$. By Proposition 5.1 in [[4],P79], it follows that

$$
\left|A\left(k_{0}+d\right)\right|=0, d=\sqrt{c}|f|_{\infty}\left|A\left(k_{0}\right)\right|^{\frac{2}{n+1}}
$$


This results that

$$
\sup _{G_{R}} v^{+} \leq k_{0}+d \leq k_{0}+C|f|_{\infty} R
$$

It remains to estimate $k_{0}$. From $k^{2}|A(k)| \leq\left\|v^{+}\right\|_{L^{2}\left(G_{R}\right)}^{2}$, one can deduce that

$$
c|A(k)|^{\frac{2}{n+1}} \leq c\left(\frac{\left\|v^{+}\right\|_{L^{2}\left(G_{R}\right)}^{2}}{k^{2}}\right)^{\frac{2}{n+1}} \leq \frac{1}{4} \text { if } k \geq k_{0}=(4 c)^{\frac{n+1}{4}}\left\|v^{+}\right\|_{L^{2}\left(G_{R}\right)}
$$

Hence,

$$
\begin{aligned}
\sup _{G_{R}} v^{+} & \leq C\left\|v^{+}\right\|_{L^{2}\left(G_{R}\right)}+c|f|_{L^{\infty}} R \\
& \leq c R^{\frac{n+1}{4}} \sup _{G_{R}} v^{+}+c|f|_{L^{\infty}} R
\end{aligned}
$$

If we choose $R$ small enough such that $c R^{\frac{n+1}{4}} \leq \frac{1}{2}$, the present lemma follows immediately from the last inequality.

Lemma 2.3. Let the assumptions in Lemma 2.2 be fulfilled and let $v \in H^{1}\left(G_{1}\right) \cap W_{l o c}^{2, l}\left(G_{1}\right), \forall l \in$ $[2, \infty)$. Moreover, $v$ satisfies $\mathscr{L}(v) \geq 0$ in $G_{1}$. Then

$$
\sup _{G_{\theta R}}|v| \leq C_{\theta}\left(\frac{1}{\left|G_{R}\right|} \int_{G_{R}} v^{2}\right)^{\frac{1}{2}}
$$

Proof. Let $\varphi(x) \in C_{c}^{\infty}\left(G_{R} \cup\left\{x_{n}=0\right\}\right)$ be the cutoff function. Then

$$
\begin{aligned}
0 & \geq \int_{G_{1} \cap\left\{x_{n} \geq \epsilon\right\}}-\varphi^{2} v^{+} \mathscr{L}(v) \\
& =\int_{G_{1} \cap\left\{x_{n} \geq \epsilon\right\}} \varphi^{2} a^{i j} \partial_{i} v^{+} \partial_{j} v^{+}+2 \int_{G_{1} \cap\left\{x_{n} \geq \epsilon\right\}} \varphi \varphi_{i} a^{i j} v^{+} \partial_{j} v^{+}+\int_{G_{1} \cap\left\{x_{n}=\epsilon\right\}} \varphi^{2} a^{i n} v^{+} \partial_{i} v^{+} \\
& +\frac{1}{2} \int_{G_{1} \cap\left\{x_{n}=\epsilon\right\}} \varphi^{2} b^{n}\left(v^{+}\right)^{2}-\sum_{j=1}^{n-1} \int_{G_{1} \cap\left\{x_{n} \geq \epsilon\right\}} \varphi^{2} b^{j} v^{+} \partial_{j} v^{+} \\
& +\frac{1}{2} \int_{G_{1} \cap\left\{x_{n} \geq \epsilon\right\}} \varphi^{2} \partial_{n} b^{n}\left(v^{+}\right)^{2}+\int_{G_{1} \cap\left\{x_{n} \geq \epsilon\right\}} \varphi \varphi_{n} b^{n}\left(v^{+}\right)^{2}
\end{aligned}
$$

Following the same arguments as in Lemma 2.2, we can select a suitable $\epsilon_{k} \rightarrow 0$ such that the first boundary integral term $\int_{G_{1} \cap\left\{x_{n}=\epsilon_{k}\right\}} \varphi^{2} a^{i n} v^{+} \partial_{i} v \rightarrow 0$ as $\epsilon_{k} \rightarrow 0$. Noting $b^{n} \geq 0$, one can get (2.22) implies that

$$
\int_{G_{1}} x_{n}\left|\varphi \partial_{x_{n}} v^{+}\right|^{2}+\left|\varphi \nabla_{x^{\prime}} v^{+}\right|^{2} d x \leq c \int_{G_{1}}\left(x_{n}\left|\partial_{n} \varphi\right|^{2}+\left|\nabla_{x^{\prime}} \varphi\right|^{2}+\varphi^{2}+\left|\partial_{n} \varphi\right|\right)\left(v^{+}\right)^{2} d x
$$

Combining the above inequality with Lemma 2.1, we get

$$
\left[\int_{G_{1}}\left(\varphi v^{+}\right)^{\frac{2(n+1)}{n-1}} d x\right]^{\frac{n-1}{n+1}} \leq C_{1} \int_{G_{1}}\left(x_{n}\left|\partial_{n} \varphi\right|^{2}+\left|\nabla_{x^{\prime}} \varphi\right|^{2}+\varphi^{2}+\left|\partial_{n} \varphi\right|\right)\left(v^{+}\right)^{2} d x
$$

For any $\theta \in(0,1)$, we construct $\gamma(\xi) \in C^{\infty}(-\infty, 1)$ with $\gamma=1$ as $\xi \leq \theta$ and $\left|\gamma^{\prime}\right| \leq c /(1-\theta)$ as $|\xi| \geq \theta, \gamma(1)=0$. Set

$$
p_{k}=\left(\frac{n+1}{n-1}\right)^{k}, \theta_{k}=\theta+\frac{1-\theta}{2^{k}}, k=0,1,2, \ldots
$$




$$
\varphi_{k}=\prod_{i=1}^{n-1} \gamma\left(\frac{2^{k+1}\left|x_{i}-\left(x_{0}\right)_{i}\right|}{\sqrt{R}}+1-2^{k+1} \theta_{k}\right) \gamma\left(\frac{2^{k+1} x_{n}}{R}+1-2^{k+1} \theta_{k}\right)
$$

From the fact that $\mathscr{L}\left(v^{+}\right) \geq 0 \Rightarrow \mathscr{L}\left(\left(v^{+}\right)^{p_{k}}\right) \geq 0$, substituting $\varphi$ and $v^{+}$in (2.23) by $\varphi_{k}$ and $\left(v^{+}\right)^{p_{k}}$ respectively, we can obtain with $G_{k}=G_{\theta_{k} R},\left.\varphi_{k}\right|_{G_{k+1}}=1$,

$$
\left(\int \varphi_{k+1}^{2}\left|v^{+}\right|^{2 p_{k+1}}\right)^{\frac{n-1}{n+1}} \leq \frac{C 4^{k+1}}{(1-\theta)^{2} R} \int \varphi_{k}^{2}\left|v^{+}\right|^{2 p_{k}}
$$

Therefor,

$$
\left\|v^{+}\right\|_{L^{2 p_{k+1}\left(G_{k+1}\right)}} \leq \frac{C}{(1-\theta)^{\sum \frac{1}{p_{k}}} R^{\sum \frac{1}{2 p_{k}}}}\left(\int_{G_{R}}\left|v^{+}\right|^{2}\right)^{\frac{1}{2}}=C(1-\theta)^{-\frac{n+1}{2}}\left(\frac{1}{R^{\frac{n+1}{2}}} \int_{G_{R}}\left|v^{+}\right|^{2}\right)^{\frac{1}{2}}
$$

This proves the present lemma.

Lemma 2.4. Let the assumptions in Lemma 2.2 be fulfilled and let $v \in W_{l o c}^{2, l}\left(G_{R}\right) \cap L^{\infty}\left(G_{R}\right), \forall l \in$ $[2, \infty)$ solves (2.5). Then for $R$ suitable small, one can have $v \in \widetilde{W}^{1,2}\left(G_{R}\right)$.

Proof. Set $\eta_{\epsilon}\left(x_{n}\right) \in C^{\infty}\left(R_{+}^{1}\right)$ that

$$
\eta_{\epsilon}\left(x_{n}\right)= \begin{cases}0, & 0<x_{n}<\epsilon \\ 1, & x_{n}>2 \epsilon\end{cases}
$$

with $\left|D^{j} \eta_{\epsilon}\right| \leq C_{j} \epsilon^{-j}$ for $x_{n} \in(\epsilon, 2 \epsilon)$. Multiplying (2.5) by $\eta_{\epsilon} v$ and integrating by parts, one can get

$$
2 \int \eta_{\epsilon} a^{i j} \partial_{i} v \partial_{j} v=\int\left(\partial_{n} \eta_{\epsilon} \partial_{j} a^{n j}-\eta_{\epsilon} \partial_{j} b^{j}-\partial_{n} \eta_{\epsilon} b^{n}\right) v^{2}+\int a^{n n} \partial_{n}^{2} \eta_{\epsilon} v^{2}-2 \int \eta_{\epsilon} f v
$$

We can choose $R$ suitable small such that

$$
\int \eta_{\epsilon} a^{i j} \partial_{i} v \partial_{j} v \geq c_{0} \int \eta_{\epsilon}\left(\left|\nabla_{x^{\prime}} v\right|^{2}+x_{n}\left|\partial_{n} v\right|^{2}\right)
$$

Selecting one term on the right side of (2.26) to estimate, one can get

$$
\left|\int a^{n n} \partial_{n}^{2} \eta_{\epsilon} v^{2}\right| \leq c|v|_{\infty}^{2} \epsilon^{-2} \int_{\epsilon}^{2 \epsilon} x_{n} d x_{n} \leq C
$$

Hence, it's easy to see that the right side of (2.26) is bounded uniformly independent of $\epsilon$. Passing $\epsilon \rightarrow 0$, one can prove the present lemma.

With the above lemmas, we can proceed to prove Theorem 1.2

The proof for Theorem 1.2 .

Fixing $x_{0} \in \partial \Omega$, without loss of generality, one can assume

$$
\partial_{x_{i}} \phi\left(x_{0}\right)=0, i=1, \ldots, n-1, \partial_{x_{n}} \phi\left(x_{0}\right)=1 .
$$

Set $s_{i}=x_{i}, i=1, \ldots, n-1, s_{n}=\phi\left(x_{1}, . ., x_{n}\right), \forall x \in B_{\delta}\left(x_{0}\right) \cap \bar{\Omega}$ for some small $\delta>0$. In the new coordinates, (1.1) transforms to

$$
\partial_{i}\left(\tilde{a}^{i j} \partial_{j} u\right)+\left(\tilde{b}^{i}-\partial_{j} \tilde{a}^{i j}\right) \partial_{i} u+f(s, u)=0 \text { in } R_{+}^{n} \cap U\left(s\left(x_{0}\right)\right)
$$


where

$$
\begin{aligned}
& \tilde{a}^{i j}=a^{i j}, i, j=1, \ldots, n-1, \tilde{a}^{i n}=a^{i j} \partial_{x_{j}} \phi, i=1, \ldots n-1, \tilde{a}^{n n}=a^{i j} \partial_{x_{i}} \phi \partial_{x_{j}} \phi, \\
& \tilde{b}^{i}=b^{i}, i=1, \ldots n-1, \tilde{b}^{n}=b^{i} \partial_{x_{i}} \phi+a^{i j} \partial_{x_{i} x_{j}} \phi, \bar{b}^{i} \triangleq \tilde{b}^{i}-\partial_{j} \tilde{a}^{i j}, \partial_{i}=\partial_{s_{i}}
\end{aligned}
$$

and $U\left(s\left(x_{0}\right)\right)$ is some neighborhood of $s\left(x_{0}\right)$, simply denoted by $U$. We now consider (2.29) in $G_{R} \subset U$ for $R \in\left(0, R_{0}\right.$ ] small. From (1.3) and (1.4), we can see $\left(\tilde{a}^{i j}\right)_{i, j \leq n-1} \geq \lambda I, a^{i n}\left(s^{\prime}, 0\right)=$ $0, i=1 \ldots, n$. Noting the condition $g(x)>0$ on the boundary and $\phi(s)=s_{n}$, one can get

$$
0<\frac{\tilde{b}^{i} \partial_{i} \phi-\partial_{j} \tilde{a}^{i j} \partial_{i} \phi}{\partial_{k} \tilde{a}^{i j} \partial_{i} \phi \partial_{j} \phi \phi^{k}}=\frac{\tilde{b}^{n}-\partial_{n} \tilde{a}^{n n}}{\partial_{n} \tilde{a}^{n n}} .
$$

By (1.3) and (2.30), it is easy to see $\partial_{n} \tilde{a}^{n n}=\partial_{n}\left(a^{i j} \partial_{x_{i}} \phi \partial_{x_{j}} \phi\right)>0$ on $s_{n}=0$ and $\bar{b}^{n}=\tilde{b}^{n}-\partial_{j} \tilde{a}^{n j}>$ 0 noting $a^{i n}\left(s^{\prime}, 0\right)=0, i=1 \ldots, n$. All the assumptions in Lemma 2.2 are fulfilled, one can get that there exists $v \in H^{1}\left(G_{R}\right) \cap W_{l o c}^{2, p}\left(G_{R}\right)$ satisfies

$$
\left\{\begin{array}{l}
\partial_{i}\left(\tilde{a}^{i j} \partial_{j} v\right)+\bar{b}^{i} \partial_{i} v+f(s, u)=0 \text { in } G_{R} \\
v=0 \text { on } \partial G_{R} \cap\left\{s_{n}>0\right\}
\end{array}\right.
$$

such that $|v|_{\infty} \leq C R$. Set $\tilde{u}=u-v$. Then $\tilde{u}$ satisfies

$$
\left\{\begin{array}{l}
\mathscr{R}(\tilde{u})=\partial_{i}\left(\tilde{a}^{i j} \partial_{j} \tilde{u}\right)+\bar{b}^{i} \partial_{i} \tilde{u}=0 \text { in } G_{R} \\
\tilde{u}=u \text { on } \partial G_{R} \cap\left\{s_{n}>0\right\}
\end{array}\right.
$$

Set $M(R)=\sup _{G_{R}} \tilde{u}$ and $m(R)=\inf _{G_{R}} \tilde{u}$ and $\underset{G_{R}}{O s c} \tilde{u}=M(R)-m(R)$. There are two cases to distinguish

$$
\text { Case } 1:\left|\left\{s \in G_{R} \mid w=2 \frac{\tilde{u}-m(R)}{M(R)-m(R)} \geq 1\right\}\right| \geq \frac{1}{2}\left|G_{R}\right|
$$

and

$$
\text { Case } 2:\left|\left\{s \in G_{R} \mid w=2 \frac{M(R)-\tilde{u}}{M(R)-m(R)} \geq 1\right\}\right| \geq \frac{1}{2}\left|G_{R}\right|
$$

Without loss of generality, we can assume Case 1 is valid. It is easy to see that

$$
\mathscr{R}(w)=0 \text { in } G_{R} \text { and } 0 \leq w \leq 2
$$

Let $\varphi \in C_{c}^{\infty}\left(G_{R} \cup\left\{x_{n}=0\right\}\right)$ with $\varphi \equiv 1$ in $G_{\theta_{1} R}$ and $\varphi \equiv 0$ in $G_{R} \backslash G_{\theta_{2} R}$ for $\left(\frac{1}{2}\right)^{\frac{2}{n+1}}<\theta_{1}<\theta_{2}<1$. For any $\epsilon>0$,

$$
\begin{aligned}
& 0=\int_{G_{R} \cap\left\{s_{n} \geq t\right\}} \varphi^{2}\left(\frac{1}{w+\epsilon}-1\right)^{+} \mathscr{R}(w) \\
& =\int_{G_{R} \cap\left\{s_{n} \geq t\right\}} \varphi^{2} \tilde{a}^{i j}\left[\left(\ln \frac{1}{w+\epsilon}\right)^{+}\right]_{i}\left[\left(\ln \frac{1}{w+\epsilon}\right)^{+}\right]_{j}+\int_{G_{R} \cap\left\{s_{n} \geq t\right\}} 2 \varphi_{i}(1-w-\epsilon)^{+} \tilde{a}^{i j}\left[\left(\ln \frac{1}{w+\epsilon}\right)^{+}\right]_{j} \\
& -\sum_{i=1}^{n-1} \int_{G_{R} \cap\left\{s_{n} \geq t\right\}} \varphi^{2}(1-w-\epsilon)^{+} \bar{b}^{i}\left[\left(\ln \frac{1}{w+\epsilon}\right)^{+}\right]_{i}-\int_{G_{R} \cap\left\{s_{n}=t\right\}} \varphi^{2}\left(\frac{1}{w+\epsilon}-1\right)^{+} \tilde{a}^{n j} \partial_{j} w \\
& -\int_{G_{R} \cap\left\{s_{n} \geq t\right\}} \varphi^{2} \bar{b}^{n} \partial_{n}\left[\left(\ln \frac{1}{w+\epsilon}\right)^{+}-(1-w-\epsilon)^{+}\right] \\
& \geq \frac{1}{2} \int_{G_{R} \cap\left\{s_{n} \geq t\right\}} \varphi^{2} \tilde{a}^{i j}\left[\left(\ln \frac{1}{w+\epsilon}\right)^{+}\right]_{i}\left[\left(\ln \frac{1}{w+\epsilon}\right)^{+}\right]_{j}
\end{aligned}
$$




$$
\begin{aligned}
& +\lim _{t \rightarrow 0} \int_{G_{R} \cap\left\{s_{n}=t\right\}} \bar{b}^{n} \varphi^{2}\left[\left(\ln \frac{1}{w+\epsilon}\right)^{+}-(1-w-\epsilon)^{+}\right] \\
& -C \int_{G_{R} \cap\left\{s_{n} \geq t\right\}} \tilde{a}^{i j} \varphi_{i} \varphi_{j}-\frac{C_{\delta}\left|G_{R}\right|}{\left(\theta_{2}-\theta_{1}\right)^{2} R}-\frac{C \delta}{R} \int_{G_{R} \cap\left\{s_{n} \geq t\right\}} \varphi^{2}\left[\left(\ln \frac{1}{w+\epsilon}\right)^{+}\right]^{2}
\end{aligned}
$$

The boundary term $\varliminf_{t \rightarrow 0} \int_{G_{R} \cap\left\{s_{n}=t\right\}} \varphi^{2} \tilde{a}^{i j}\left(\frac{1}{w+\epsilon}-1\right)^{+} \partial_{j} w=0$ follows from Lemma 2.4

$$
v \in H^{1}\left(G_{R}\right), u \in \widetilde{W}^{1,2}\left(G_{R}\right) \Rightarrow w \in \widetilde{W}^{1,2}\left(G_{R}\right)
$$

This implies that $\int_{G_{R}} s_{n}\left|\partial_{j} w\right|^{2} \leq C_{R}$. If $\varliminf_{t \rightarrow 0} \int_{G_{R} \cap\left\{s_{n}=t\right\}} s_{n}^{2}\left|\partial_{j} w\right|^{2} \geq c>0$, one can get

$$
\int_{G_{R}} s_{n}\left|\partial_{j} w\right|^{2} \geq \int_{0}^{R} c / s_{n} d s_{n}=\infty
$$

which is a contradiction. Hence, for fixed $\epsilon>0$,

$$
\varliminf_{t \rightarrow 0} \int_{G_{R} \cap\left\{s_{n}=t\right\}} \varphi^{2} \tilde{a}^{i j}\left(\frac{1}{w+\epsilon}-1\right)^{+} \partial_{j} w \leq C_{R, \epsilon} \varliminf_{t \rightarrow 0}\left(\int_{G_{R} \cap\left\{s_{n}=t\right\}} s_{n}^{2}\left|\partial_{j} w\right|^{2}\right)^{\frac{1}{2}}=0
$$

Therefor, from (2.36) and $\bar{b}^{n}>0$,

$$
\begin{aligned}
& \left.\int_{G_{\theta_{1} R}} s_{n}\left|\partial_{n}\left(\ln \frac{1}{w+\epsilon}\right)^{+}\right|^{2}+\left|\nabla_{s^{\prime}}\left(\ln \frac{1}{w+\epsilon}\right)^{+}\right|^{2}\right) \\
\leq & C \int_{G_{R}}\left(s_{n} \varphi_{s_{n}}^{2}+\left|\nabla_{s^{\prime}} \varphi\right|^{2}\right)+\frac{C_{\delta}\left|G_{R}\right|}{\left(\theta_{2}-\theta_{1}\right)^{2} R}+\frac{C \delta}{R} \int_{G_{\theta_{2} R}}\left[\left(\ln \frac{1}{w+\epsilon}\right)^{+}\right]^{2} \\
\leq & \frac{C_{\delta}\left|G_{R}\right|}{\left(\theta_{2}-\theta_{1}\right)^{2} R}+\frac{C \delta}{R} \int_{G_{\theta_{2} R}}\left[\left(\ln \frac{1}{w+\epsilon}\right)^{+}\right]^{2}
\end{aligned}
$$

for some constant $C$ independent of $\epsilon$. In view of Case 1 it follows

$$
\begin{aligned}
\left|\left\{(s, t) \in G_{\theta_{1} R} \mid\left(\ln \frac{1}{w+\epsilon}\right)^{+}=0\right\}\right| & \geq\left|\left\{(s, t) \in G_{R} \mid w+\epsilon \geq 1\right\}\right|-\left|G_{R} \backslash G_{\theta_{1} R}\right| \\
& \geq\left(\theta_{1}^{\frac{n+1}{2}}-\frac{1}{2}\right)\left|G_{R}\right| \geq \epsilon_{0}\left|G_{R}\right|
\end{aligned}
$$

Hence Lemma 2.1 implies

$$
\begin{aligned}
& \int_{G_{\theta_{1} R}}\left[\left(\ln \frac{1}{w+\epsilon}\right)^{+}\right]^{2} \\
\leq & C R \int_{G_{\theta_{1} R}} s_{n}\left|\partial_{s_{n}}\left(\ln \frac{1}{w+\epsilon}\right)^{+}\right|^{2}+\left|\nabla_{s^{\prime}}\left(\ln \frac{1}{w+\epsilon}\right)^{+}\right|^{2} \\
\leq & \frac{C_{\delta}\left|G_{R}\right|}{\left(\theta_{2}-\theta_{1}\right)^{2}}+C \delta \int_{G_{\theta_{2} R}}\left[\left(\ln \frac{1}{w+\epsilon}\right)^{+}\right]^{2}
\end{aligned}
$$

Set $\delta=\frac{1}{2 C}$. Then

$$
\int_{G_{\theta_{1} R}}\left|\left(\ln \frac{1}{w+\epsilon}\right)^{+}\right|^{2} d x \leq \frac{C\left|G_{R}\right|}{\left(1-\theta_{1}\right)^{2}}
$$


As $\mathscr{R}(w+\epsilon)=0 \Rightarrow \mathscr{R}\left(\left(\ln \frac{1}{w+\epsilon}\right)^{+}\right) \geq 0$, by Lemma 2.3 .

$$
\sup _{G_{\theta R}}\left(\ln \frac{1}{w+\epsilon}\right)^{+} \leq C_{\theta}\left(\frac{1}{\left|G_{R}\right|} \int_{G_{R}}\left|\left(\ln \frac{1}{w+\epsilon}\right)^{+}\right|^{2}\right)^{\frac{1}{2}} \leq C_{2}
$$

for some constant independent of $\epsilon$. Therefore

$$
\inf _{G_{\theta R}}(w+\epsilon) \geq e^{-C_{2}} \quad \forall \epsilon>0
$$

and by the definition of $w$ we have

$$
m(\theta R)-m(R) \geq \frac{1}{C}(M(R)-m(R))
$$

Adding $M(\theta R)-m(\theta R)$ to the above inequality and rearranging again soon yield

$$
\underset{G_{\theta R}}{O s c} \tilde{u} \leq\left(1-\frac{1}{C}\right) \underset{G_{R}}{O s c} \tilde{u} \text { for all } R \in\left(0, R_{0}\right]
$$

for some constant $C$ independent of $\epsilon$. From a standard argument it turns out

$$
\underset{G_{\rho}}{O s c} \tilde{u} \leq C\left(\frac{\rho}{R}\right)^{\beta} \underset{G_{R}}{O s c} \tilde{u} \text { for all } 0<\rho \leq R
$$

for some constant $\beta<1$ under control. This implies that

$$
\underset{G_{\rho}}{O s c} u \leq c_{1}\left(\frac{\rho}{R}\right)^{\beta}+c_{2} R \leq c \rho^{\frac{\beta}{1+\beta}}
$$

if we set $\rho=R^{1+\frac{1}{\beta}}$. This proves Theorem 1.2 ,

\section{Higher regularities to the boundary for a specific equation}

As one can see, we have proved $C^{\alpha}$ estimates on the boundary for a general form of the degenerate elliptic equations. But for a special form of the degenerate elliptic equation, we will have more regularities which are also needed for our later analysis. At first, we shall give some notations and results about the regularity of solutions to some degenerate elliptic equations due to $[8$.

Define $I_{q}(v)$ and $I_{\beta}(v)$ by:

$$
\begin{gathered}
I_{q}(v)=\left\|y \partial_{y y} v\right\|_{L^{q}\left(R_{+}^{n}\right)}+\left\|\Lambda_{1}^{2} v\right\|_{L^{q}\left(R_{+}^{n}\right)}+\left\|y^{\frac{1}{2}} \Lambda_{1} v_{y}\right\|_{L^{q}\left(R_{+}^{n}\right)}+\left\|v_{y}\right\|_{L^{q}\left(R_{+}^{n}\right)}+\|v\|_{L^{q}\left(R_{+}^{n}\right)}, \\
I_{\beta}(v)=\left[y \partial_{y y} v\right]_{\dot{C}^{\beta}\left(\overline{R_{+}^{n}}\right)}+\left[\Lambda_{1}^{2} v\right]_{\dot{C}^{\beta}\left(\overline{R_{+}^{n}}\right)}+\left[y^{\frac{1}{2}} \Lambda_{1} v_{y}\right]_{\dot{C}^{\beta}\left(\overline{R_{+}^{n}}\right)}+\left[v_{y}\right]_{\dot{C}^{\beta}\left(\overline{R_{+}^{n}}\right)}+\|v\|_{L^{\infty}\left(R_{+}^{n}\right)},
\end{gathered}
$$

where $\Lambda_{1}$ is a singular integral operator with the symbol $\sigma\left(\Lambda_{1}\right)=|\xi|$. Also we say a function $v(x, y)$ in $\dot{C}^{\alpha}\left(\overline{R_{+}^{n}}\right), \alpha \in R_{+}^{1} \backslash Z$, if

$$
|v|_{\dot{C}^{\alpha}\left(\overline{R_{+}^{n}}\right)}=\sum_{|\beta| \leq[\alpha]}\left|D^{\beta} v\right|_{C\left(\overline{R_{+}^{n}}\right)}+[v]_{\dot{C}^{\alpha}\left(\overline{R_{+}^{n}}\right)}<\infty,
$$

where

$$
[v]_{\dot{C}^{\alpha}\left(\overline{R_{+}^{n}}\right)}=\sum_{|\beta|=[\alpha]} \sup _{y \geq 0, x \neq \bar{x} \in R^{n-1}}\left(\frac{\left|D_{x}^{\beta} v(x, y)-D_{x}^{\beta} v(\bar{x}, y)\right|}{|x-\bar{x}|^{\alpha}}\right) .
$$


Set

$$
\begin{aligned}
\hat{K}(y, s, \xi) & =\hat{K}_{1}(y, s, \xi)+\hat{K}_{2}(y, s, \xi) \\
\hat{K}_{1}(y, s, \xi) & =\varphi_{1}\left(|\xi|^{2} y\right) \varphi_{2}\left(|\xi|^{2} s\right)\left(|\xi|^{2} s\right)^{a-1} \chi_{(y,+\infty)}(s) \\
\hat{K}_{2}(y, s, \xi) & =\varphi_{2}\left(|\xi|^{2} y\right) \varphi_{1}\left(|\xi|^{2} s\right)\left(|\xi|^{2} s\right)^{a-1} \chi_{(0, y)}(s) \\
\varphi_{1}(t) & =t^{\frac{1-a}{2}} I_{a-1}(2 \sqrt{t}), \varphi_{2}(t)=t^{\frac{1-a}{2}} K_{a-1}(2 \sqrt{t}),
\end{aligned}
$$

where $I_{a-1}(t), K_{a-1}(t)$ satisfy the equation

$$
t^{2} w_{t t}+t w_{t}-\left((a-1)^{2}+t^{2}\right) w=0
$$

Define an operator $K$ as the following:

$$
K(f)(x, y)=\int_{0}^{+\infty} K(y, s, \cdot) * f(s, \cdot) \mathrm{d} s
$$

Suppose $f \in L^{p}\left(R_{+}^{n}\right)$ has compact support in $\overline{R_{+}^{n}}$ and $a>\frac{3}{2}$, we will have

$$
K(f) \rightarrow 0 \text { as }(x, y) \rightarrow \infty \text {, moreover, } I_{p}(K(f)) \leq C\|f\|_{L^{p}, p} \geq 2
$$

The proof of (3.7) is due to [8. See Theorem 1.2, Lemma 5.2 and Lemma 5.3 in [8]. Also we list some other lemmas in 8 for later use.

Let $\psi \in C_{c}^{\infty}\left(\overline{R_{+}^{n}}\right)$ be a cutoff function with $\psi(x, y)=1$ as $|x| \leq 1 / 2, y \leq 1 / 2$ and $\psi=0$ as $|x| \geq 1$ or $|y| \geq 1$. Set $\psi_{r}(x, y)=\psi\left(\frac{x}{r}, \frac{y}{r}\right)$.

Lemma 3.1. (Lemma 5.4 in [8]) Suppose that $u \in C^{2}\left(R_{+}^{n}\right) \cap L^{p}\left(R_{+}^{n}\right)$ with $u_{x}$,yuy $\in L^{p}\left(R_{+}^{n}\right)$ satisfies

$$
L(u)=y u_{y y}+\sum_{i, j} a_{i j} u_{x_{i} x_{j}}+y \sum_{j} a_{j} u_{y x_{j}}+\sum_{j} b_{j} u_{x_{j}}+b u_{y}=f, \text { in } R_{+}^{n},
$$

where $a_{i j}, a_{j}, b_{j}, b$ are all in $C\left(\overline{R_{+}^{n}}\right)$ with $a_{i j}(0)=\delta_{i j}, b(0)>\frac{3}{2}, f \in L^{\infty}\left(R_{+}^{n}\right)$ and that for some $\epsilon>0$,

$$
\lim _{y \rightarrow 0} y^{b(0)-1-\epsilon} u(x, y)=0 \text { uniformly for all } x \in R^{n-1} .
$$

Then for sufficiently large $p$, there are $r=r(p)>0$ such that

$$
I_{p}\left(\psi_{r} u\right) \leq C_{r},
$$

for some constant depending only on $p,\left\|\psi_{2 r} f\right\|_{L^{p}},\left\|\psi_{2 r} u\right\|_{L^{p}},\left\|\psi_{2 r} u_{x}\right\|_{L^{p}}$ and $\left\|y \psi_{2 r} u_{y}\right\|_{L^{p}}$ provided that $p>n$ or $p>\frac{n}{2}$ and $b(0)-2-\epsilon>0$.

Lemma 3.2. (Lemma 5.5 in [8]) Suppose that $w, \partial_{x} w, y \partial_{y} w \in \dot{C}_{l o c}^{\alpha}\left(\overline{R_{+}^{n}}\right) \cap C^{2}\left(R_{+}^{n}\right)$ with $\alpha \in R_{+}^{1} \backslash Z$ and $w$ satisfies (3.8), where $a_{i j}, a_{j}, b_{j}, b, f$ are all in $\dot{C}_{l o c}^{\alpha}\left(\overline{R_{+}^{n}}\right)$ with $a_{i j}(0)=\delta_{i j}, b(0)>\frac{3}{2}$. Then

$$
I_{\alpha}\left(\psi_{r} w\right) \leq C,
$$

for some positive constants $r$ and $C$, depending on $\alpha,\left|\psi_{2 r} f\right|_{\alpha},\left|\psi_{2 r} w\right|_{\alpha},\left|\psi_{2 r} \partial_{x} w\right|_{\alpha}$ and $\left|y \psi_{2 r} \partial_{y} w\right|_{\alpha}$.

Denote by $W_{\alpha}^{1, p}(U)$ the completion of the space of all the functions $u$ in $C^{1}(\bar{U})$ under the norm

$$
\left(\int_{U} y^{p \alpha}|D u|^{p} d x d y+\int_{U} y^{p \alpha}|u|^{p} d x d y\right)^{\frac{1}{p}} .
$$

Here we always assume $U \subset R_{+}^{n}$, bounded and $\partial U \cap\{y=0\}$ nonempty. 
Lemma 3.3. (Lemma 8.3 in [8] Appendix B) Let $U \in C^{1}$ be bounded domain and let $\alpha \in(0,1)$, $p>1 /(1-\alpha)$. Then the following maps are continuous

$$
\begin{aligned}
& W_{\alpha}^{1, p}(U) \quad C^{\beta}(\bar{U}) \text { where } \beta=1-\alpha-\frac{n}{p}, \text { if } p>\frac{n}{(1-\alpha)}, \\
& W_{\alpha}^{1, p}(U) \quad \hookrightarrow L^{q}(U) \text { where } q<\frac{n p}{n-(1-\alpha) p}, \text { if } p<\frac{n}{(1-\alpha)} .
\end{aligned}
$$

Denote by $\bar{W}^{2, p}\left(\overline{R_{+}^{n}}\right)$ the completion of the space of all the functions $u \in C_{c}^{\infty}\left(\overline{R_{+}^{n}}\right)$ under the norm $I_{p}(u)$.

Lemma 3.4. If $u \in L_{l o c}^{\infty}\left(\overline{R_{+}^{n}}\right)$ is a nonnegative solution of (1.8) with $a>\frac{3}{2}, \alpha \geq 1$, we must have $u \in \bar{W}_{l o c}^{2,2}\left(\overline{R_{+}^{n}}\right)$.

Proof. By the similar arguments as we did in Lemma 2.4 one can see that $u \in \widetilde{W}_{l o c}^{1,2}\left(\overline{R_{+}^{n}}\right)$. Let $\psi(x, y) \in C_{c}^{\infty}\left(\overline{R_{+}^{n}}\right)$ be a cutoff function and $\psi_{r}(x, y)=\psi\left(\frac{x}{\sqrt{r}}, \frac{y}{r}\right)$. Set $u_{r}=\psi_{r} u$. Then $u_{r}$ satisfies that

$$
\begin{aligned}
& y \partial_{y y} u_{r}+a \partial_{y} u_{r}+\Delta_{x} u_{r} \\
= & 2 y \partial_{y} \psi_{r} \partial_{y} u+a \partial_{y} \psi_{r} u+2 \nabla_{x} \psi_{r} \nabla_{x} u+y \partial_{y y} \psi_{r} u+\Delta_{x} \psi_{r} u-\psi_{r} u^{\alpha}=f
\end{aligned}
$$

Set $v=K(f)$ where $K(f)$ is defined in (3.6). As $f \in L^{2}\left(R_{+}^{n}\right)$ with compact support, it is valid that $I_{2}(v) \leq C$ by (3.7). It follows that for $w=u_{r}-v$, there holds

$$
y \partial_{y}^{2} w+a \partial_{y} w+\Delta_{x} w=0 \text { in } R_{+}^{n}
$$

Multiplying the above equation by $-\psi_{R}^{2} w$ and integrating by parts, one can see

$$
\begin{aligned}
& \int_{R_{x}^{n-1} \times\{y \geq \epsilon\}}\left(y \psi_{R}^{2}\left(\partial_{y} w\right)^{2}+\psi_{R}^{2}\left|\nabla_{x} w\right|^{2}\right)+\frac{a-1}{2} \int_{R_{x}^{n-1} \times\{y=\epsilon\}} \psi_{R}^{2} w^{2} \\
=\quad & -\int_{R_{x}^{n-1} \times\{y=\epsilon\}} \epsilon \psi_{R}^{2} w \partial_{y} w-2 \int_{R_{x}^{n-1} \times\{y \geq \epsilon\}} y \psi_{R} w \partial_{y} \psi_{R} \partial_{y} w \\
& -(a-1) \int_{R_{x}^{n-1} \times\{y \geq \epsilon\}} \partial_{y} \psi_{R} \psi_{R} w^{2}-2 \int_{R_{x}^{n-1} \times\{y \geq \epsilon\}} \nabla_{x} \psi_{R} \cdot \nabla_{x} w \psi_{R} w \\
\leq \quad & -\int_{R_{x}^{n-1} \times\{y=\epsilon\}} \epsilon \psi_{R}^{2} w \partial_{y} w+\frac{1}{2} \int_{R_{x}^{n-1} \times\{y \geq \epsilon\}} y \psi_{R}^{2}\left(\partial_{y} w\right)^{2} \\
& +\frac{1}{2} \int_{R_{x}^{n-1} \times\{y \geq \epsilon\}} \psi_{R}^{2}\left|\nabla_{x} w\right|^{2}+\frac{C}{R} \int_{R_{+}^{n}} w^{2}
\end{aligned}
$$

The remaining thing is to prove

$$
\varliminf_{\epsilon \rightarrow 0} \int_{R_{x}^{n-1} \times\{y=\epsilon\}} \epsilon \psi_{R}^{2} w \partial_{y} w=0
$$

Without loss of generality, it can be assumed that $\|v(x, 0)\|_{L^{2}\left(R_{x}^{n-1}\right)} \leq c$. Hence,

$$
\begin{aligned}
& v(x, y)=\int_{0}^{y} \partial_{y} v d t+v(x, 0) \\
\Rightarrow \quad & \|v(x, \epsilon)\|_{L^{2}\left(R_{x}^{n-1}\right)} \leq c \sqrt{\epsilon}\left\|v_{y}\right\|_{L^{2}\left(R_{+}^{n}\right)}+2\|v(x, 0)\|_{L^{2}\left(R_{x}^{n-1}\right)}
\end{aligned}
$$

Noting $\left|u_{r}\right|_{\infty} \leq c$, one can get

$$
\int_{R_{x}^{n-1} \times\{y=\epsilon\}} \psi_{R}^{2} w^{2} \leq c \text { independent of } \epsilon
$$


Also, as $y^{\frac{1}{2}} \partial_{y} w \in L^{2}\left(R_{x}^{n-1} \times(0,1)\right)$, it follows from the same arguments in Lemma 2.5 that

$$
\varliminf_{\epsilon \rightarrow 0} \int_{R_{x}^{n-1} \times\{y=\epsilon\}} \epsilon^{2} \psi_{R}^{2}\left|\partial_{y} w\right|^{2}=0
$$

Combining (3.20) with (3.21), one can see (3.18) is valid. Choosing a suitable subsequence of $\epsilon_{k} \rightarrow 0$ in 3.17) yields that

$$
\int_{R^{n}} y \psi_{R}^{2}\left(\partial_{y} w\right)^{2}+\psi_{R}^{2}\left|\nabla_{x} w\right|^{2} \leq \frac{C}{R} \int_{R_{+}^{n}} w^{2}
$$

Passing $R \rightarrow \infty$ in (3.22), one can get $D_{x, y} w \equiv 0$ which means $w \equiv$ const. From the compact support of $u_{r}$ and the asymptotic property of $v, w$ must vanish in $R_{+}^{n}$. This completes the proof of the present lemma.

With the aid of Lemma 3.4, we can now prove Theorem 1.3.

The proof for Theorem 1.3. by Lemma 3.4 Lemma 2.1 and Sobolev embedding theorem, one can get

$$
y \partial_{y} u_{r} \in H^{1}\left(R_{+}^{n}\right), \nabla_{x} u_{r} \in \widetilde{W}^{1,2}\left(R_{+}^{n}\right) \Rightarrow y \partial_{y} u_{r}, \nabla_{x} u_{r} \in L^{\theta}\left(R_{+}^{n}\right), \theta=\frac{2(n+1)}{n-1}
$$

Set $v=K(f)$. It follows from (3.7) that $v \in \bar{W}^{2,2}\left(R_{+}^{n}\right) \cap \bar{W}^{2, \theta}\left(R_{+}^{n}\right)$. By the same arguments as in Lemma [3.4, we can get $u_{r}=v \in \bar{W}^{2,2}\left(R_{+}^{n}\right) \cap \bar{W}^{2, \theta}\left(R_{+}^{n}\right)$ with $\nabla_{x} u_{r} \in W_{\frac{1}{2}}^{1, \theta}\left(R_{+}^{n}\right)$. Again, by Lemma 3.3 and Sobolev embedding theorem, it yields that

$$
y \partial_{y} u_{r}, \nabla_{x} u_{r} \in L^{\frac{n \theta}{n-\frac{\theta}{2}}}\left(R_{+}^{n}\right), \partial_{y} u_{r} \in L^{\theta}\left(R_{+}^{n}\right)
$$

Set $\theta_{k}=\frac{n \theta_{k-1}}{n-\frac{\theta_{k-1}}{2}}, \theta_{0}=\theta$. Repeating the above arguments, one can get

$$
y \partial_{y} u_{r}, \nabla_{x} u_{r} \in L^{\theta_{k}}\left(R_{+}^{n}\right), \partial_{y} u_{r} \in L^{\theta_{k-1}}\left(R_{+}^{n}\right), k=1,2 \ldots
$$

Choosing $k$ so large such that $\theta_{k}>2 n$, we must have

$$
v \in \bar{W}^{2,2}\left(R_{+}^{n}\right) \cap \bar{W}^{2, \theta_{k}}\left(R_{+}^{n}\right), y \partial_{y} u_{r}, \nabla_{x} u_{r}, u_{r} \in C^{\beta}\left(\overline{R_{+}^{n}}\right), \gamma=\frac{1}{2}-\frac{n}{\theta_{k}}
$$

Now we can apply Lemma 3.2 to $u_{r}$, we can get $I_{\gamma}\left(u_{r}\right) \leq C$. This means

$$
y \partial_{y}^{2} u_{r}, \Lambda_{1}^{2} u_{r}, y^{\frac{1}{2}} \partial_{y} \Lambda_{1} u_{r}, \partial_{y} u_{r} \in \dot{C}^{\gamma}\left(\overline{R_{+}^{n}}\right) .
$$

Applying Lemma 3.2 to $\Lambda_{1} u_{r}$ yields that $I_{\gamma}\left(\Lambda_{1} u_{r}\right) \leq C$, or

$$
y \partial_{y}^{2} \Lambda_{1} u_{r}, \Lambda_{1}^{3} u_{r}, \partial_{y} \Lambda_{1} u_{r}, y^{\frac{1}{2}} \partial_{y} \Lambda_{1}^{2} u_{r} \in \dot{C}^{\gamma}\left(\overline{R_{+}^{n}}\right)
$$

Applying Lemma 3.2 to $\partial_{y} u_{r}$ again, one can get

$$
y \partial_{y}^{3} u_{r}, \Lambda_{1}^{2} \partial_{y} u_{r}, \partial_{y}^{2} u_{r}, y^{\frac{1}{2}} \partial_{y}^{2} \Lambda_{1} u_{r} \in \dot{C}^{\gamma}\left(\overline{R_{+}^{n}}\right) .
$$

This implies that $u_{r} \in C^{2}\left(\overline{R_{+}^{n}}\right)$. Now we have finished the proof of Theorem 1.3 .

Remark 3.1. As we already know $u \in C^{2}\left(\overline{R_{+}^{n}}\right)$, by the maximum principle in [9], we have $u>$ $0 \in C^{2}\left(\overline{R_{+}^{n}}\right)$. Proceeding the above arguments and by induction, we can get $u \in C^{\infty}\left(\overline{R_{+}^{n}}\right)$. 


\section{The proof of a priori bounds}

Before proving the main theorem, we introduce two Liouville type lemmas concerning the semi-linear elliptic equations.

Lemma 4.1. Let $u \geq 0$ be a $C^{2}$ solution of

$$
\Delta u+u^{\alpha}=0 \text { in } R^{n}
$$

Then $u \equiv 0$, for $1 \leq \alpha<\frac{n+2}{n-2}$.

For the proof of Lemma 4.1, we refer to [5] and [1]. Another Lemma concerns the degenerate case.

Lemma 4.2. Let $u(x, y) \geq 0$ be a $C^{2}\left(\overline{R_{+}^{n}}\right)$ solution of

$$
y u_{y y}+a u_{y}+\Delta u+u^{\alpha}=0 \text { in } R_{+}^{n}
$$

with $a>1$. Then $u \equiv 0$ for $1 \leq \alpha<\frac{n+2 a+1}{n+2 a-3}$.

This lemma was proved in author's another paper 9 .

With all the above preparations, we are now in a position to prove Theorem 1.1. We shall use the blow up method to prove Theorem 1.1. If Theorem 1.1 is not valid, we may assume $\left|u^{k}\right|_{\infty}=M_{k} \rightarrow \infty$ as $k \rightarrow \infty$. Hence one can choose $P_{k} \in \Omega$ such that $u\left(P_{k}\right) \geq \frac{M_{k}}{2}$. There are two cases we shall distinguish with.

Case 1. $d\left(P_{k}, \partial \Omega\right) \geq \delta>0$. This is the non-degenerate case and it is easy to deal with. In fact the final blow-up function satisfies

$$
\Delta u+u^{\alpha}=0 \text { in } R^{n}, u(0) \geq \frac{1}{2}
$$

By Lemma 4.1, we get a contradiction. For details, we refer to 5 and 9 .

Case 2. $d\left(P_{k}, \partial \Omega\right) \rightarrow 0$ as $k \rightarrow \infty . \lim _{k \rightarrow \infty} P_{k}=P \in \partial \Omega$. Without loss of generality, we may assume $\partial_{i} \phi(P)=0, i=1, \ldots, n-1, \partial_{n} \phi \neq 0$ and $P$ to be the origin. Let $s_{i}=x_{i}, i=1, . ., n-1, s_{n}=$ $\phi\left(x_{1}, \ldots, x_{n}\right)$ in $B_{\delta}(0) \cap \Omega$. Equation (1.1) can be transformed to

$$
\tilde{a}^{i j} \partial_{i j} u^{k}+\tilde{b}^{i} \partial_{i} u^{k}+f\left(s, u^{k}\right)=0 \text { in } B_{\delta^{\prime}}(0) \cap\left\{s_{n}>0\right\} .
$$

The coefficients are defined the same as in (2.30). Set

$$
\lambda_{k}^{\frac{1}{\alpha-1}} M_{k}=1, p_{i}=\frac{s_{i}-s_{i}^{P_{k}}}{\sqrt{\lambda_{k}}}, i=1, \ldots n-1, p_{n}=\frac{s_{n}-s_{n}^{P_{k}}}{\lambda_{k}}, v^{k}(p)=\lambda_{k}^{\frac{1}{\alpha-1}} u^{k}(s)
$$

Then $v^{k}$ should satisfy, in $H_{k}=B_{\frac{\delta^{\prime}}{2 \sqrt{\lambda_{k}}}}(0) \cap\left\{p_{n}>-\frac{s_{n}^{P_{k}}}{\lambda_{k}}\right\}$

$$
\begin{aligned}
\lambda_{k}^{-1} \tilde{a}^{n n} \partial_{n}^{2} v^{k} & +2 \sum_{i=1}^{n} \lambda_{k}^{-\frac{1}{2}} \tilde{a}^{i n} \partial_{i n} v^{k}+\sum_{i, j=1}^{n-1} \tilde{a}^{i j} \partial_{i j} v^{k} \\
& +\sum_{i=1}^{n-1} \lambda_{k}^{\frac{1}{2}} \tilde{b}^{i} \partial_{i} v^{k}+\tilde{b}^{n} \partial_{n} v^{k}+\lambda_{k}^{\frac{\alpha}{\alpha-1}} f\left(p, \lambda_{k}^{-\frac{1}{\alpha-1}} v^{k}\right)=0
\end{aligned}
$$

Then we will have the following lemma. 
Lemma 4.3. In the region $H_{k}$ considered, one has

$$
\begin{aligned}
& \left(\tilde{a}^{i j}\right) \geq c_{0} I, \text { for } 1 \leq i, j \leq n-1, c_{0}>0 \\
& \tilde{a}^{i n}=A^{i n}(p) \lambda_{k}\left(p_{n}+\frac{s_{n}^{P_{k}}}{\lambda_{k}}\right) \text { for } i=1, \ldots, n
\end{aligned}
$$

where

$$
A^{i n}(p) \in C^{1}\left(\bar{H}_{k}\right), A^{n n}\left(p^{\prime},-\frac{s_{n}^{P_{k}}}{\lambda_{k}}\right)>0, \frac{\tilde{b}^{n}\left(p^{\prime},-\frac{s_{n}^{P_{k}}}{\lambda_{k}}\right)}{A^{n n}\left(p^{\prime},-\frac{s_{n}^{P_{k}}}{\lambda_{k}}\right)}>\frac{3}{2}, p^{\prime}=\left(p_{1}, \ldots, p_{n-1}\right) .
$$

Proof. Noting $\tilde{a}^{n n}=a^{i j} \partial_{i} \phi \partial_{j} \phi=0$ on $\left\{s_{n}=0\right\}$, we get

$$
\begin{aligned}
\tilde{a}^{n n}(\bar{s}) & =\int_{0}^{1} \frac{d\left(a^{i j} \partial_{i} \phi \partial_{j} \phi\right)\left(\bar{s}^{\prime}, t \bar{s}_{n}\right)}{d t} d t=\bar{s}_{n} \int_{0}^{1} \partial_{s_{n}}\left(a^{i j} \partial_{i} \phi \partial_{j} \phi\right)\left(\bar{s}^{\prime}, t \bar{s}_{n}\right) d t \\
& =A^{n n} \lambda_{k}\left(\bar{p}_{n}+\frac{s_{n}^{P_{k}}}{\lambda_{k}}\right), \text { where } A^{n n}=\int_{0}^{1} \partial_{s_{n}}\left(a^{i j} \partial_{i} \phi \partial_{j} \phi\right)\left(\bar{s}^{\prime}, t \bar{s}_{n}\right) d t
\end{aligned}
$$

From (1.3), we see that $\nabla\left(\tilde{a}^{n n}\right) \neq 0$ on $\left\{s_{n}=0\right\}$ which implies that $\partial_{s_{n}} \tilde{a}^{n n}\left(s^{\prime}, 0\right)>0$ or $A^{n n}\left(p^{\prime},-\frac{s_{n}^{P_{k}}}{\lambda_{k}}\right)>0$ in the region considered. The $C^{1}$ property of $A^{n n}$ follows from the $C^{2}$ property of $a^{i j}, \phi$ immediately. The last term in (4.5) follows from (1.6).

By Lemma 4.3. (4.2) changes to

$$
\begin{aligned}
A^{n n}\left(p_{n}+\frac{s_{n}^{P_{k}}}{\lambda_{k}}\right) \partial_{n}^{2} v^{k} & +2 \sum_{i=1}^{n-1} \lambda_{k}^{\frac{1}{2}} A^{i n}\left(p_{n}+\frac{s_{n}^{P_{k}}}{\lambda_{k}}\right) \partial_{i n} v^{k}+\sum_{i, j=1}^{n-1} \tilde{a}^{i j} \partial_{i j} v^{k} \\
& +\sum_{i=1}^{n-1} \lambda_{k}^{\frac{1}{2}} \tilde{b}^{i} \partial_{i} v^{k}+\tilde{b}^{n} \partial_{n} v^{k}+\lambda_{k}^{\frac{\alpha}{\alpha-1}} f\left(p, \lambda_{k}^{-\frac{1}{\alpha-1}} v^{k}\right)=0
\end{aligned}
$$

We must take care of the limit of $\frac{s_{n}^{P_{k}}}{\lambda_{k}}$.

$\underline{\text { Case 2.1: }}: \frac{s_{n}^{P_{k}}}{\lambda_{k}} \rightarrow \infty$. Set $q_{i}=p_{i}, q_{n}=2 \sqrt{p_{n}+s_{n}^{P_{k}} \lambda_{k}^{-1}}-2 \sqrt{s_{n}^{P_{k}} \lambda_{k}^{-1}}$. Then with $v^{k}(0) \geq \frac{1}{2}$

$$
\begin{aligned}
\partial_{n}^{2} v^{k} & +2 \sum_{i=1}^{n-1} \bar{A}^{i n}\left(\lambda_{k}^{\frac{1}{2}} q_{n}+\sqrt{s_{n}^{P_{k}}}\right) \partial_{i n} v^{k}+\sum_{i, j=1}^{n-1} \bar{a}^{i j} \partial_{i j} v^{k} \\
& +\sum_{i=1}^{n-1} \lambda_{k}^{\frac{1}{2}} \bar{b}^{i} \partial_{i} v^{k}+\frac{2 \bar{b}^{n}}{q_{n}+2 \sqrt{s_{n}^{P_{k}} \lambda_{k}^{-1}}} \partial_{n} v^{k}+\lambda_{k}^{\frac{\alpha}{\alpha-1}} \bar{f}\left(q, \lambda_{k}^{-\frac{1}{\alpha-1}} v^{k}\right)=0, \text { in } J_{k}
\end{aligned}
$$

where

$$
\bar{A}^{i n}=\frac{A^{i n}}{A^{n n}}, \bar{a}^{i j}=\frac{\tilde{a}^{i j}}{A^{n n}}, \bar{b}^{i}=\frac{\tilde{b}^{i}}{A^{n n}}, \bar{f}=\frac{f}{A^{n n}}
$$

It is necessary to show $J_{k}$ can be chosen arbitrary large as $k \rightarrow \infty$. Since $p \in H_{k}$, it follows that

$$
\begin{aligned}
& \left|q^{\prime}\right|^{2}+\left[\left(\frac{q_{n}}{2}+\sqrt{\frac{s_{n}^{P_{k}}}{\lambda_{k}}}\right)^{2}-\frac{s_{n}^{P_{k}}}{\lambda_{k}}\right]^{2}<\frac{\delta^{\prime 2}}{4 \lambda_{k}} \Longleftrightarrow\left|q^{\prime}\right|^{2}+q_{n}^{2}\left(\frac{q_{n}}{4}+\sqrt{\frac{s_{n}^{P_{k}}}{\lambda_{k}}}\right)^{2}<\frac{\delta^{\prime 2}}{4 \lambda_{k}} \\
& \Rightarrow q_{n}<\frac{\delta^{\prime}}{\sqrt{s_{n}^{P_{k}}}} \text { noting that } q_{n}>-2 \sqrt{\frac{s_{n}^{P_{k}}}{\lambda_{k}}} .
\end{aligned}
$$


From $s_{n}^{P_{k}} \rightarrow 0$ and $\frac{s_{n}^{P_{k}}}{\lambda_{k}} \rightarrow \infty$, one can get

$$
\frac{\delta^{\prime 2}}{4 \lambda_{k}}=4 l_{k}^{2} \max ^{2}\left\{\frac{\delta^{\prime}}{4 \sqrt{s_{n}^{P_{k}}}}, \sqrt{\frac{s_{n}^{P_{k}}}{\lambda_{k}}}\right\}, l_{k} \rightarrow \infty \text { as } k \rightarrow \infty .
$$

Since

$$
\left|q^{\prime}\right|^{2}+q_{n}^{2}\left(\frac{q_{n}}{4}+\sqrt{\frac{s_{n}^{P_{k}}}{\lambda_{k}}}\right)^{2} \leq\left|q^{\prime}\right|^{2}+4 \max ^{2}\left\{\frac{\delta^{\prime}}{4 \sqrt{s_{n}^{P_{k}}}}, \sqrt{\frac{s_{n}^{P_{k}}}{\lambda_{k}}}\right\} q_{n}^{2}
$$

we can take $J_{k}=B_{l_{k}}(0) \cap\left\{q_{n}>-2 \sqrt{\frac{s_{n}^{P_{k}}}{\lambda_{k}}}\right\}$. For any fixed $R>0, \exists k_{R}$, for $k \geq k_{R}$, one has $B_{R}(0) \subset J_{k}$. Hence, (4.8) is valid in $B_{R}(0)$ for $k \geq k_{R}$. As $\lambda I \leq\left\{\bar{a}^{i j}\right\} \leq \Lambda I$ and $s_{n}^{P_{k}}, \lambda_{k} \rightarrow 0$, (4.8) is uniformly elliptic in $B_{R}(0)$. Noting

$$
\left|\lambda_{k}^{\frac{1}{2}} \bar{b}^{i}\right|_{C^{0}\left(B_{R}(0)\right)}+\left|\frac{2 \bar{b}^{n}}{q_{n}+2 \sqrt{s_{n}^{P_{k}} \lambda_{k}^{-1}}}\right|_{C^{0}\left(B_{R}(0)\right)}+\left|\lambda_{k}^{\frac{\alpha}{\alpha-1}} \bar{f}\left(q, \lambda^{-\frac{1}{\alpha-1}} v^{k}\right)\right|_{\infty} \leq C \text { independent of } k,
$$

by standard elliptic equation theorem, one can get $\left\|v^{k}\right\|_{W^{2, p}\left(B_{R}(0)\right)} \leq C \Rightarrow\left\|v^{k}\right\|_{C^{1, \beta}\left(\overline{B_{R}(0)}\right)} \leq C$ uniformly, with $\beta=1-\frac{n}{p}$. Thus, passing $k \rightarrow \infty$, we get the limit equation

$$
\Delta v+v^{\alpha}=0 \text { in } R^{n}, v(0) \geq \frac{1}{2}, \alpha<\frac{n+2 a+1}{n+2 a-3}<\frac{n+2}{n-2}
$$

By Lemma 4.1, the above equation only admits trivial solution which is a contradiction to $v(0) \geq \frac{1}{2}$

$\underline{\text { Case 2.2: }}: \frac{s_{n}}{\lambda_{k}} \rightarrow c_{0}<\infty$. Set $q_{i}=p_{i}, i=1, \ldots, n-1, q_{n}=p_{n}+\frac{s_{n}^{P_{k}}}{\lambda_{k}}$. Then (4.7) transforms to in $B_{\frac{\delta^{\prime}}{2 \sqrt{\lambda_{k}}}}\left(\left(0^{\prime}, \frac{s_{n}^{P_{k}}}{\lambda_{k}}\right)\right) \cap R_{+}^{n}$ with $v^{k}\left(0^{\prime}, \frac{s_{n}^{P_{k}}}{\lambda_{k}}\right) \geq \frac{1}{2}$,

$$
\begin{aligned}
A^{n n} q_{n} \partial_{n}^{2} v^{k} & +2 \sum_{i=1}^{n-1} \lambda_{k}^{\frac{1}{2}} A^{i n} q_{n} \partial_{i n} v^{k}+\sum_{i, j=1}^{n-1} \tilde{a}^{i j} \partial_{i j} v^{k} \\
& +\sum_{i=1}^{n-1} \lambda_{k}^{\frac{1}{2}} \tilde{b}^{i} \partial_{i} v^{k}+\tilde{b}^{n} \partial_{n} v^{k}+\lambda_{k}^{\frac{\alpha}{\alpha-1}} f\left(q, \lambda_{k}^{-\frac{1}{\alpha-1}} v^{k}\right)=0
\end{aligned}
$$

As $\frac{s_{n}^{P_{k}}}{\lambda_{k}}$ is uniformly bounded, we may assume (4.13) is satisfied in $B_{\frac{\delta^{\prime}}{4 \sqrt{\lambda_{k}}}}(0) \cap R_{+}^{n}$.

(a): $c_{0}>0$, by choosing $\delta$ small enough, 4.13) is uniformly elliptic in $B_{\delta}\left(0^{\prime}, c_{0}\right)$, hence,

$$
\left\|v^{k}\right\|_{W^{2, p}\left(B_{\delta}\left(0^{\prime}, c_{0}\right)\right)} \leq C \Rightarrow\left\|v^{k}\right\|_{C^{1, \beta}\left(\overline{B_{\delta}\left(0^{\prime}, c_{0}\right)}\right)} \leq C .
$$

(b): $c_{0}=0$, by Theorem 1.2 one can see that

$$
\left|v^{k}\left(0^{\prime}, \frac{s_{n}^{P_{k}}}{\lambda_{k}}\right)-v^{k}(0)\right| \leq C\left(\frac{s_{n}^{P_{k}}}{\lambda_{k}}\right)^{\alpha}
$$

Since $\frac{s_{n}^{P_{k}}}{\lambda_{k}} \rightarrow 0$ as $k \rightarrow \infty$, it follows that $v^{k}(0) \geq \frac{1}{4}$ for $k$ large enough. Again by Theorem 1.2 one can get $v^{k}(q) \geq \frac{1}{8}, \forall q \in B_{\tilde{\delta}}(0) \cap R_{+}^{n}$ for some $\tilde{\delta}$ small.

Therefor, by the above arguments, passing the limit $k \rightarrow \infty$, one can get

$$
v\left(0, c_{0}\right)=\lim _{k \rightarrow \infty} v^{k}\left(0^{\prime}, \frac{s_{n}^{P_{k}}}{\lambda_{k}}\right) \geq \frac{1}{2}, c_{0}>0
$$


or,

$$
v(q) \geq \frac{1}{8}, \forall q \in B_{\tilde{\delta}(0)} \cap R_{+}^{n}, c_{0}=0
$$

and $v$ satisfies

$$
A^{n n}(P) q_{n} \partial_{n}^{2} v+\sum_{i, j=1}^{n-1} \tilde{a}^{i j}(P) \partial_{i j} v+\tilde{b}^{n}(P) \partial_{n} v+h(P) v^{\alpha}=0 \text { in } R_{+}^{n}
$$

where $\frac{\tilde{b}^{n}(P)}{A^{n n}(P)}>\frac{3}{2}$ by $(1.6)$. By a rotating and a stretching of the coordinates, we have that

$$
q_{n} \partial_{n}^{2} v+\Delta_{n-1} v+\bar{b} \partial_{n} v+v^{\alpha}=0 \text { in } R_{+}^{n}, a \geq \bar{b}>\frac{3}{2}
$$

Since $\alpha<\frac{n+2 a+1}{n+2 a-3} \leq \frac{n+2 \bar{b}+1}{n+2 b-3}$, by Lemma 4.2 the above equation admits only trivial solution which is a contradiction. This completes the proof of Theorem 1.1

\section{References}

[1] L.Caffarelli, B.Gidas, J.Spruck, Asymptotic symmetry and local behavior of semilinear elliptic with critical Sobolev growth, Comm.Pure Appl.Math., 42(1989), pp.271-297.

[2] G.Fichera, Sulle equazioni differenziali lineari ellittico-paraboliche del secondo ordine, Atti Accad.Naz.Lincei.Mem.Cl.Sci.Fis.Mat.Nat.Sez.I(8) 5 (1956), 1-30.

[3] G.Fichera, "On a unified theory of boundary value problems for elliptic-parabolic equations of second order," in Boundary problems. Differential equations, Univ. of Wisconsin Press, Madison, Wis., 1960, pp.97-120.

[4] M.Giaquinta, Introduction to regularity theory for nonlinear elliptic systems, Lectures in Mathematics ETH Zürich. Birkhäuser Verlag, Basel, 1993

[5] B.Gidas, J.Spruck, Global and local behavior of positive solutions of nonlinear elliptic equations, Comm. Pure Appl. Math. 34(1981), no.4, 525-598.

[6] B.Gidas, J.Spruck, A priori bounds for positive solutions of nonlinear elliptic equations. Comm. Partial Differential Equations 6 (1981), no. 8, 883-901.

[7] J.X.Hong, On boundary value problems for mixed equations with characteristic degenerate surfaces, Chin. Ann. of Math., 1981, Vol.2, no.4, 407-424.

[8] J.X.Hong, G.G.Huang, $L^{p}$ and Hölder estimates for a class of degenerate elliptic partial differential equations and its applications, Int Math Res Notices (2012) Vol.2012 Issue 13 2889-2941

[9] G.G.Huang, A Liouville theorem of degenerate elliptic equation and its application,http://arxiv.org/abs/1211.2520

[10] M.V.Keldy $\check{s}$, On certain cases of degeneration of equations of elliptic type on the boundary of a domain, Dokl.Akad.Nauk SSSR 77(1951),181-183.

[11] Oleinik, O. A.; Radkevic, E. V. Second order equations with nonnegative characteristic form. Translated from the Russian by Paul C. Fife. Plenum Press, New York-London, 1973. 